\title{
EXOTIC LEFT-ORDERINGS OF THE FREE GROUPS FROM THE DEHORNOY ORDERING
}

\author{
ADAM CLAY \\ (Received 2 November 2010)
}

\begin{abstract}
We show that the restriction of the Dehornoy ordering to an appropriate free subgroup of the three-strand braid group defines a left-ordering of the free group on $k$ generators, $k>1$, that has no convex subgroups.
\end{abstract}

2010 Mathematics subject classification: primary 20F36; secondary 20F60, $20 \mathrm{E} 05$.

Keywords and phrases: left-orderable groups, free groups, braid groups.

\section{Introduction}

A group $G$ is said to be left-orderable if there exists a strict total ordering of its elements such that $g<h$ implies $f g<f h$ for all $f, g, h$ in $G$. To each left-ordering < of a group $G$, we can associate the set $P=\{g \in G \mid g>1\}$, which is called the positive cone associated to the left-ordering $<$. The positive cone $P$ satisfies $P \cdot P \subset P$, and $P \sqcup P^{-1} \sqcup\{1\}=G$. Conversely, any subset $P$ satisfying these two properties defines a strict total ordering of the elements of $G$ via $g<h$ if and only if $g^{-1} h \in P$. Any ordering defined in this way is easily seen to be invariant under left multiplication.

We may strengthen our conditions on a left-ordering $<$ of $G$ by requiring that, for all $g, h>1$ in $G$, there must exist a positive integer $n$ such that $g<h g^{n}$. In this case, the ordering is called Conradian (after the work of Conrad in [2]). It has since been observed that, equivalently, we may ask that this condition hold for $n=2$ [9].

Finally, the strongest condition we may require of an ordering $<$ of $G$ is that the ordering be invariant under multiplication from both sides, that is, $g<h$ implies $f g<f h$ and $g f<h f$ for all $f, g, h$ in $G$. Equivalently, we may require that the positive cone associated to the ordering $<$ of $G$ be preserved by conjugation. If either of these equivalent conditions is satisfied by the ordering $<$ of $G$, then the ordering is said to be a bi-ordering.

An important structure associated to a given left-ordering $<$ of $G$ is the set of convex subgroups of $G$. A subgroup $H \subset G$ is said to be convex in $G$ (with respect to the ordering $<$ ) if whenever $f, h$ are in $H$ and $g$ is in $G$, the implication $f<g<h \Rightarrow g \in H$ holds.

(C) 2011 Australian Mathematical Publishing Association Inc. 0004-9727/2011 \$16.00 
Owing to work of Conrad and Hölder, the convex subgroups of bi-orderings and Conradian orderings are very well understood [2]. This leaves us with understanding the set of convex subgroups for the case of left-orderings that are neither bi-orderings nor Conradian orderings. This problem seems to be quite difficult, as constructing Conradian orderings and bi-orderings of a group $G$ is in general somewhat easier than constructing left-orderings of a group that are not Conradian orderings.

Two of the primary methods for constructing non-Conradian orderings of a group $G$ are given by the following proposition and theorem.

PROPOSITION 1.1. Let $G$ be a group, $K$ a subgroup of $G$ left-ordered by the ordering $\prec$, and $G / K$ the set of left cosets of $K$ in $G$. Suppose that $G / K$ is ordered by the ordering $\prec^{\prime}$, satisfying $g K \prec^{\prime} h K \Rightarrow f g K \prec^{\prime} f h K$ for all $f, g, h$ in $G$. Then a leftordering $<$ can be defined on $G$, according to the rule that, for every $g$ in $G, 1<g$ if $g \in K$ and $1 \prec g$, or if $g \notin K$ and $K \prec^{\prime} g K$.

TheOREM 1.2 (Conrad [2]). A group $G$ is left-orderable if and only if $G$ acts effectively by order-preserving automorphisms on a linearly ordered set.

In both of these cases, at least some of the convex subgroups of the constructed ordering are obvious. In Proposition 1.1, the subgroup $K$ is a convex subgroup in the left-ordering < of $G$. In Theorem 1.2, the stabilizers under the $G$-action of points in the given linearly ordered set correspond to convex subgroups (see [2] or [9] for details of the construction). In light of the fact that both of these known methods for producing left-orderings of a group result in a left-ordering that (often) admits convex subgroups, it is quite surprising to find that some non-Conradian left-orderings may contain no proper, nontrivial convex subgroups whatsoever. In this paper, we will left-order the free groups of finite rank in such a way that the free group contains no proper, nontrivial convex subgroups with respect to our constructed ordering. The construction relies heavily on the Dehornoy ordering of the braid group $B_{3}$.

The existence and a construction of such orderings of the free groups seems to have appeared only in [7]. The construction there, unlike our present setting, deals with creating a very unusual effective action on the rationals. Our present approach is in simpler algebraic terms.

It is also worth noting that admitting a Conradian or bi-ordering that has no proper, nontrivial convex subgroups is a very restrictive condition on the group $G$, as the following theorem shows.

THEOREM 1.3 [2]. Suppose that G admits a Conradian or bi-ordering which has no proper, nontrivial convex subgroups. Then $G$ is a subgroup of $(\mathbb{R},+)$.

In the case where $G$ admits a non-Conradian left-ordering having no proper, nontrivial convex subgroups, it is not likely that the structure of $G$ must be so restricted. While we will see that free groups admit such left-orderings, there are also nonfree, non-abelian groups that admit such left-orderings as well [1, Example 7.2.3]. It has also recently been shown that the braid groups themselves admit many left-orderings with no convex subgroups; see [10]. 


\section{A left-ordering of $\boldsymbol{F}_{2}$ having no convex subgroups}

As a warm-up for the general case, which will be slightly more involved, we deal first with the free group on two generators.

We begin by defining the Dehornoy left-ordering of the braid groups (also known as the 'standard' ordering), whose positive cone we shall denote $P_{D}[3,4]$. Recall that for each integer $n \geq 2$, the Artin braid group $B_{n}$ is the group generated by $\sigma_{1}, \sigma_{2}, \ldots, \sigma_{n-1}$, subject to the relations

$$
\sigma_{i} \sigma_{j}=\sigma_{j} \sigma_{i} \text { if }|i-j|>1, \quad \sigma_{i} \sigma_{j} \sigma_{i}=\sigma_{j} \sigma_{i} \sigma_{j} \text { if }|i-j|=1 .
$$

Definition 2.1. Let $w$ be a word in the generators $\sigma_{i}, \ldots, \sigma_{n-1}$. Then $w$ is said to be $i$-positive if the generator $\sigma_{i}$ occurs in $W$ with only positive exponents, $i$-negative if $\sigma_{i}$ occurs with only negative exponents, and $i$-neutral if $\sigma_{i}$ does not occur in $w$.

We then define the positive cone of the Dehornoy ordering as follows.

Definition 2.2. The positive cone $P_{D} \subset B_{n}$ of the Dehornoy ordering is the set

$$
P_{D}=\left\{\beta \in B_{n}: \beta \text { is } i \text {-positive for some } i \leq n-1\right\} .
$$

Let $\beta \in B_{n}$ be any braid. An extremely important property of this ordering is that the conjugate $\beta \sigma_{k} \beta^{-1}$ is always $i$-positive for some $i$, for every generator $\sigma_{k}$ in $B_{n}$. This property is referred to as the subword property [4].

Recall that the commutator subgroup $\left[B_{3}, B_{3}\right]$ is isomorphic to the free group $F_{2}$ on two generators. The commutator subgroup is generated by the braids $\beta_{1}=\sigma_{2} \sigma_{1}^{-1}$ and $\beta_{2}=\sigma_{1} \sigma_{2} \sigma_{1}^{-2}$ [8]. Of course we can instead take as free generators the 1-positive braids $\beta_{1}^{-1}=\sigma_{1} \sigma_{2}^{-1}$ and $\beta_{2}^{-1} \beta_{1}^{-1}=\sigma_{1}^{2} \sigma_{2}^{-2}$, since any generating set of $F_{2}$ with only two elements will freely generate $F_{2}$.

Define a positive cone $P \subset F_{2}$ by $P=\left[B_{3}, B_{3}\right] \cap P_{D}$, with associated ordering < of $F_{2}$. Thus, the left-ordering $<$ of $F_{2}$ is the restriction of the Dehornoy ordering $<_{D}$ of $B_{3}$ to the (free) commutator subgroup $\left[B_{3}, B_{3}\right]$.

THEOREM 2.3. The ordering $<$ of $F_{2}$ has no proper, nontrivial convex subgroups.

ProOF. Let $C \subset F_{2}=\left[B_{3}, B_{3}\right]$ be a nontrivial convex subgroup. Then we may choose $1<\beta \in F_{2}$ that is 1-positive (no nontrivial 1-neutral braids lie in $\left[B_{3}, B_{3}\right]$, because they do not have zero total exponent).

There are now two cases to consider.

Case 1. Suppose that $\beta$ commutes with $\sigma_{2}$. Then $\beta=\Delta_{3}^{2 p} \sigma_{2}^{q}$ for some integers $p, q$ ([5], here $\Delta=\sigma_{1} \sigma_{2} \sigma_{1}$ ). Since $\beta \in\left[B_{3}, B_{3}\right]$, we know that $q=-6 p$, since $\beta$ must have zero total exponent, and $p>0$ because we have chosen $\beta$ to be 1-positive. Then we have that $\Delta_{3}^{2}<\Delta_{3}^{4 p} \sigma_{2}^{-12 p}=\beta^{2}$, so that $\langle\beta\rangle$ is cofinal in the Dehornoy ordering [4]. Therefore, there exist integers $k, l$ such that in $F_{2}$ we have

$$
1<\sigma_{1} \sigma_{2}^{-1}<\beta^{k} \text { and } 1<\sigma_{1}^{2} \sigma_{2}^{-2}<\beta^{l},
$$


and thus $\sigma_{1} \sigma_{2}^{-1}, \sigma_{1}^{2} \sigma_{2}^{-2} \in C$ by convexity. Therefore we must have $C=F_{2}$, as $C$ contains both generators of $F_{2}$.

Case 2. Suppose that $\beta$ and $\sigma_{2}$ do not commute. Let $k>0$, and observe that $\beta \sigma_{2}^{k} \beta^{-1}$ is a 1-positive braid by the subword property, so that the commutator $\beta \sigma_{2}^{k} \beta^{-1} \sigma_{2}^{-k}$ is also 1-positive. Next, because $\beta$ is 1-positive, the braid $\sigma_{2}^{k} \beta^{-1} \sigma_{2}^{-k}$ is 1-negative, so that $\sigma_{2}^{k} \beta^{-1} \sigma_{2}^{-k}<1$, and thus $\beta \sigma_{2}^{k} \beta^{-1} \sigma_{2}^{-k}<\beta$. Thus, we have shown that $1<$ $\beta \sigma_{2}^{k} \beta^{-1} \sigma_{2}^{-k}<\beta$, so that $\beta \sigma_{2}^{k} \beta^{-1} \sigma_{2}^{-k}$ must lie in the subgroup $C$, by convexity.

Now both the braids $\beta$ and $\beta \sigma_{2}^{k} \beta^{-1} \sigma_{2}^{-k}$ lie in the convex subgroup $C$, so the braid $\sigma_{2}^{k} \beta^{-1} \sigma_{2}^{-k}$ (and hence its inverse $\sigma_{2}^{k} \beta \sigma_{2}^{-k}$ ) must also lie in $C$, for any choice of positive integer $k$.

We now refine our choice of braid $\beta \in C$. Suppose that $\beta$ is represented by the 1-positive braid word $\sigma_{2}^{u} \sigma_{1} w$, where $u$ is any integer, and $w$ is a 1-positive, 1-neutral or empty word. Choose $k>0$ so that $u^{\prime}=k+u>0$, and set $\beta^{\prime}=\sigma_{2}^{k} \beta \sigma_{2}^{-k}$, so that $\beta^{\prime}$ is represented by the 1-positive braid word $\sigma_{2}^{u^{\prime}} \sigma_{1} w \sigma_{2}^{-k}$. Note that $\beta^{\prime} \in C$, from our work above.

We will now show that $C$ must contain both generators of $F_{2}$. Observe that the braid represented by the word $\sigma_{2} \sigma_{1}^{-1} \sigma_{2}^{u^{\prime}} \sigma_{1} w \sigma_{2}^{-k}$ is 1-positive, as $\sigma_{2}\left(\sigma_{1}^{-1} \sigma_{2}^{u^{\prime}} \sigma_{1}\right) w \sigma_{2}^{-k}=$ $\sigma_{2}\left(\sigma_{2} \sigma_{1}^{u^{\prime}} \sigma_{2}^{-1}\right) w \sigma_{2}^{-k}$, and $u^{\prime}>0$. Therefore

$$
1<\sigma_{2} \sigma_{1}^{-1} \sigma_{2}^{u^{\prime}} \sigma_{1} w \sigma_{2}^{-k} \Rightarrow \sigma_{1} \sigma_{2}^{-1}<\sigma_{2}^{u^{\prime}} \sigma_{1} w \sigma_{2}^{-k}=\beta^{\prime} \in C
$$

and since $1<\sigma_{1} \sigma_{2}^{-1}$, this implies that $\sigma_{1} \sigma_{2}^{-1} \in C$ by convexity.

Considering the second generator $\sigma_{1}^{2} \sigma_{2}^{-2}$, observe that the braid represented by the word $\sigma_{2}^{2} \sigma_{1}^{-2} \sigma_{2}^{u^{\prime}} \sigma_{1} w \sigma_{2}^{-k}$ is 1-positive, as we compute

$$
\sigma_{2}^{2} \sigma_{1}^{-1}\left(\sigma_{1}^{-1} \sigma_{2}^{u^{\prime}} \sigma_{1}\right) w \sigma_{2}^{-k}=\sigma_{2}^{2} \sigma_{1}^{-1}\left(\sigma_{2} \sigma_{1}^{u^{\prime}} \sigma_{2}^{-1}\right) w \sigma_{2}^{-k}
$$

and

$$
\sigma_{2}^{2}\left(\sigma_{1}^{-1} \sigma_{2} \sigma_{1}\right) \sigma_{1}^{u^{\prime}-1} \sigma_{2}^{-1} w \sigma_{2}^{-k}=\sigma_{2}^{2}\left(\sigma_{2} \sigma_{1} \sigma_{2}^{-1}\right) \sigma_{1}^{u^{\prime}-1} \sigma_{2}^{-1} w \sigma_{2}^{-k},
$$

where $u^{\prime}>0$. Therefore

$$
1<\sigma_{2}^{2} \sigma_{1}^{-2} \sigma_{2}^{u^{\prime}} \sigma_{1} w \sigma_{2}^{-k} \Rightarrow \sigma_{1}^{2} \sigma_{2}^{-2}<\sigma_{2}^{u^{\prime}} \sigma_{1} w \sigma_{2}^{-k}=\beta^{\prime} \in C,
$$

and since $1<\sigma_{1}^{2} \sigma_{2}^{-2}$, we conclude from convexity of $C$ that $\sigma_{1}^{2} \sigma_{2}^{-2} \in C$.

Thus, $C$ contains both generators of $F_{2}$, so that $C=F_{2}$.

\section{Left-ordering the free groups of rank greater than two}

We now extend our results to cover those free groups $F_{k}$ with $k>2$. Let $x=\sigma_{1} \sigma_{2}^{-1}$ and $y=\sigma_{1}^{2} \sigma_{2}^{-2}$ denote the generators of $F_{2}$, and we let $K_{n}$ denote the kernel of the map $F_{2} \rightarrow \mathbb{Z}_{n-1}$ defined by $y \mapsto 0, x \mapsto 1$. Here $\mathbb{Z}_{n-1}$ is the cyclic group 
of order $n-1$. We will employ a proof very similar to that of Theorem 2.3, by considering $K_{n} \subset F_{2}=\left[B_{3}, B_{3}\right]$, and showing that the restriction of the Dehornoy ordering to $K_{n}$ has no convex subgroups. First we need to find a generating set for $K_{n}$.

LEMMA 3.1. The subgroup $K_{n}$ is free of rank $n$, with basis

$$
y, x^{n-1}, x y x^{n-2}, x^{2} y x^{n-3}, \ldots, x^{n-2} y x .
$$

PROOF. From [11, Lemma 7.56] we know that $K_{n}$ is finitely generated. Moreover, we may compute a generating set of $K_{n}$ as follows: consider the generating set $g_{1}=$ $x, g_{2}=x^{-1}, g_{3}=y, g_{4}=y^{-1}$ of $F_{2}$, and let $1, x, x^{2}, \ldots, x^{n-2}$ be representatives of the right cosets of $K_{n} \subset F_{2}$. For all $i, j$, there exist $h_{i j}$ and some coset representative $x^{k(i, j)}$ such that we may write $x^{i} g_{j}=h_{i j} x^{k(i, j)}$. The elements $h_{i j}$ form a generating set for $K_{n}$.

In our present setting, for $i<n-2$ we get

$$
x^{i} g_{1}=x^{i} \cdot x=1 \cdot x^{i+1},
$$

so that $h(i, 1)=1$, and for $i=n-2$ we get $h(i, 1)=x^{n-1}$. Similarly, we compute for $i \geq 1$ that

$$
x^{i} g_{2}=x^{i} \cdot x^{-1}=1 \cdot x^{i-1},
$$

so that $h(i, 2)=1$, and for $i=0$ we compute $h(i, 2)=x^{-(n-1)}$.

Next, for all $i$, we compute

$$
x^{i} y^{ \pm 1}=x^{i} y^{ \pm 1} x^{-i} \cdot x^{i}
$$

so that $h(i, 3)=h(i, 4)^{-1}=x^{i} y x^{-i}$. Eliminating inverses from this generating set yields the set

$$
y, x^{n-1}, x y x^{-1}, x^{2} y x^{-2}, \ldots, x^{n-2} y x^{-(n-2)} .
$$

From [6, Proposition 3.9] we deduce that $K_{n}$ is of rank $n$, and therefore the generating set above must provide a basis for $K_{n}$. Right-multiplying those generators of the form $x^{i} y x^{-i}$ by the generator $x^{n-1}$ yields the desired generating set.

Also important in the proof of Theorem 2.3 was the action of conjugation by $\sigma_{2}$. In order to generalize our theorem, we must make the following analysis.

Let $F_{2}$ be the free group on two generators $x$ and $y$, and define an automorphism $\phi: F_{2} \rightarrow F_{2}$ according to the formulas $\phi(x)=x y^{-1} x$ and $\phi(y)=x y^{-1} x^{2}$. Then the following lemma holds.

LEMMA 3.2. Consider $F_{2}$ as the commutator subgroup $\left[B_{3}, B_{3}\right]$ with generators $x=$ $\sigma_{1} \sigma_{2}^{-1}$ and $y=\sigma_{1}^{2} \sigma_{2}^{-2}$. Then the automorphism $\phi$ of $F_{2}$ corresponds to conjugation of $\left[B_{3}, B_{3}\right]$ by the generator $\sigma_{2} \in B_{3}$, so that $\phi(g)=\sigma_{2}^{-1} g \sigma_{2}$ for all $g \in F_{2}$. 
PROOF. The proof is computational. First conjugating the generator $x$, we compute

$$
\begin{aligned}
\phi(x) & =x y^{-1} x \\
& =\sigma_{1} \sigma_{2}^{-1} \sigma_{2}^{2} \sigma_{1}^{-2} \sigma_{1} \sigma_{2}^{-1} \\
& =\left(\sigma_{1} \sigma_{2} \sigma_{1}^{-1}\right) \sigma_{2}^{-1} \\
& =\left(\sigma_{2}^{-1} \sigma_{1} \sigma_{2}\right) \sigma_{2}^{-1} \\
& =\sigma_{2}^{-1} \sigma_{1} \sigma_{2}^{-1} \sigma_{2} \\
& =\sigma_{2}^{-1} x \sigma_{2}
\end{aligned}
$$

and

$$
\begin{aligned}
\phi(y) & =x y^{-1} x^{2} \\
& =\sigma_{1} \sigma_{2}^{-1} \sigma_{2}^{2} \sigma_{1}^{-2} \sigma_{1} \sigma_{2}^{-1} \sigma_{1} \sigma_{2}^{-1} \\
& =\left(\sigma_{1} \sigma_{2} \sigma_{1}^{-1}\right) \sigma_{2}^{-1} \sigma_{1} \sigma_{2}^{-1} \\
& =\left(\sigma_{2}^{-1} \sigma_{1} \sigma_{2}\right) \sigma_{2}^{-1} \sigma_{1} \sigma_{2}^{-1} \\
& =\sigma_{2}^{-1} \sigma_{1}^{2} \sigma_{2}^{-1} \\
& =\sigma_{2}^{-1} \sigma_{1}^{2} \sigma_{2}^{-2} \sigma_{2} \\
& =\sigma_{2}^{-1} y \sigma_{2} .
\end{aligned}
$$

This concludes the proof.

This computation allows us to show that $K_{n}$ is fixed by the conjugation action of $\sigma_{2}^{6}$ or $\sigma_{2}^{-6}$ on the commutator subgroup $\left[B_{3}, B_{3}\right]$.

LEMMA 3.3. Let $\phi: F_{2} \rightarrow F_{2}$ be the map arising from conjugation of $\left[B_{3}, B_{3}\right]$ by $\sigma_{2}$, namely $\phi(x)=x y^{-1} x$ and $\phi(y)=x y^{-1} x^{2}$. Then, for all $n, \phi^{6}\left(K_{n}\right)=K_{n}$.

PROOF. Consider the abelianization $F_{2} \stackrel{a b}{\longrightarrow} \mathbb{Z} \oplus \mathbb{Z}$. We find that $\phi$ descends to a map $\phi_{*}: \mathbb{Z} \oplus \mathbb{Z} \rightarrow \mathbb{Z} \oplus \mathbb{Z}$, and that relative to the basis $\{a b(x), a b(y)\}$ the map $\phi_{*}$ is represented by the matrix

$$
\left(\begin{array}{rr}
2 & 3 \\
-1 & -1
\end{array}\right) \text {. }
$$

The sixth power of this matrix is the identity. It follows that for any normal subgroup $K$ such that $F_{2} / K$ is abelian, we have $\phi^{6}(K)=K$.

Lastly, note that any generator of $K_{n}$, when we substitute $x=\sigma_{1} \sigma_{2}^{-1}$ and $y=$ $\sigma_{1}^{2} \sigma_{2}^{-2}$, yields a product of braid group generators of the form

$$
\sigma_{1}^{l_{1}} \sigma_{2}^{k_{1}} \sigma_{1}^{l_{2}} \cdots \sigma_{2}^{k_{m-1}} \sigma_{1}^{l_{m}} \sigma_{2}^{k_{m}}
$$

where $k_{i}<0$ and $l_{i}>0$ for all $i$. Therefore, we require the following lemma in order to compare the generators to different braids in $K_{n}$. 
LEMMA 3.4. Any braid represented by a word of the form

$$
\sigma_{2}^{k_{1}} \sigma_{1}^{l_{1}} \cdots \sigma_{2}^{k_{m}} \sigma_{1}^{l_{m}} \sigma_{2}^{n} \sigma_{1}
$$

where $k_{i}>0, l_{i}<0$ for all $i$, and $n>1$, is 1-positive.

PROOF. We use induction on $m$, the length of the product. For $m=0$, the claim is trivial. Assuming that the claim holds for those products of length $m-1$, we use the identities $\sigma_{1}^{k} \sigma_{2} \sigma_{1}=\sigma_{2} \sigma_{1} \sigma_{2}^{k}$ and $\sigma_{1}^{-1} \sigma_{2}^{k} \sigma_{1}=\sigma_{2} \sigma_{1}^{k} \sigma_{2}^{-1}$, and compute that

$$
\begin{aligned}
\sigma_{2}^{k_{1}} \sigma_{1}^{l_{2}} \cdots \sigma_{2}^{k_{m}} \sigma_{1}^{l_{m}} \sigma_{2}^{n} \sigma_{1} & =\sigma_{2}^{k_{1}} \sigma_{1}^{l_{2}} \cdots \sigma_{2}^{k_{m}} \sigma_{1}^{l_{m}+1}\left(\sigma_{1}^{-1} \sigma_{2}^{n} \sigma_{1}\right) \\
& =\sigma_{2}^{k_{1}} \sigma_{1}^{l_{2}} \cdots \sigma_{2}^{k_{m}} \sigma_{1}^{l_{m}+1}\left(\sigma_{2} \sigma_{1}^{n} \sigma_{2}^{-1}\right) \\
& =\sigma_{2}^{k_{1}} \sigma_{1}^{l_{2}} \cdots \sigma_{2}^{k_{m}}\left(\sigma_{1}^{l_{m}+1} \sigma_{2} \sigma_{1}\right) \sigma_{1}^{n-1} \sigma_{2}^{-1} \\
& =\sigma_{2}^{k_{1}} \sigma_{1}^{l_{2}} \cdots \sigma_{2}^{k_{m}}\left(\sigma_{2} \sigma_{1} \sigma_{2}^{l_{m}+1}\right) \sigma_{1}^{n-1} \sigma_{2}^{-1} \\
& =\sigma_{2}^{k_{1}} \sigma_{1}^{l_{2}} \cdots \sigma_{2}^{k_{m}+1} \sigma_{1}\left(\sigma_{2}^{l_{m}+1} \sigma_{1}^{n-1} \sigma_{2}^{-1}\right) .
\end{aligned}
$$

The bracketed expression $\sigma_{2}^{l_{m}+1} \sigma_{1}^{n-1} \sigma_{2}^{-1}$ is 1 -positive as $n>1$, and the remaining terms in the product above are representative of a 1-positive braid, by assumption. By induction, the claim is proven.

THEOREM 3.5. Let $n>2$. Then the restriction of the Dehornoy ordering to the subgroup $K_{n} \subset F_{2}=\left[B_{3}, B_{3}\right]$ has no proper, nontrivial convex subgroups.

PROOF. We proceed similarly to Theorem 2.3. Suppose that $C \subset K_{n}$ is a nontrivial, convex subgroup, and let $\beta \in C$ be a 1-positive braid. Denote the generators of $K_{n}$ by $g_{1}, g_{2}, \ldots, g_{n}$; from Lemma 3.1 we know that $g_{i}>1$ for all $i$. There are two cases to consider.

Case 1. The braid $\beta$ commutes with $\sigma_{2}$. In this case, we proceed as in Case 1 of Theorem 2.3, to conclude that $\langle\beta\rangle$ must be cofinal in the Dehornoy ordering. Thus, we can find an integer $k$ such that $\beta^{k}>g_{i}>1$ for every generator $g_{i}$ of $K_{n}$. Then $g_{i} \in C$ for all $i$, and we conclude that $C=K_{n}$.

Case 2. Suppose that $\beta$ and $\sigma_{2}$ do not commute, and we proceed as in Case 2 of Theorem 2.3. Then, by the subword property of the Dehornoy ordering, we know that $\beta \sigma_{2}^{k} \beta^{-1}>1$ for all $k>0$, and hence $\beta \sigma_{2}^{k} \beta^{-1} \sigma_{2}^{-k}>1$ as well. We deduce that $1<\beta \sigma_{2}^{k} \beta^{-1} \sigma_{2}^{-k}<\beta$ for all $k>0$ as before. However, the braid $\beta \sigma_{2}^{k} \beta^{-1} \sigma_{2}^{-k}$ is not necessarily an element of $K_{n}$, but as conjugation by $\sigma_{2}^{6}$ preserves $K_{n}$ by Lemma 3.3, we have $\beta \sigma_{2}^{6 k} \beta^{-1} \sigma_{2}^{-6 k} \in K_{n}$ for all $k>0$. Hence, the inequality $1<\beta \sigma_{2}^{k} \beta^{-1} \sigma_{2}^{-k}<$ $\beta$ yields $\beta \sigma_{2}^{6 k} \beta^{-1} \sigma_{2}^{-6 k} \in C$ for all $k>0$. We conclude that $\sigma_{2}^{6 k} \beta^{-1} \sigma_{2}^{-6 k} \in C$ for all $k>0$.

Proceeding as in the proof of Theorem 2.3, we may conjugate $\beta$ by an appropriate (sixth) power of $\sigma_{2}$ to conclude that the convex subgroup $C$ in $K_{n}$ contains a braid 
represented by a word of the form $\sigma_{2}^{u} \sigma_{1} w$, where $u>1$, and $w$ is a 1-positive, 1-neutral or empty word. Then for each generator $g_{i}$ of $K_{n}$, consider the braid represented by the word $g_{i}^{-1} \sigma_{2}^{u} \sigma_{1} w$. As each $g_{i}$ contains only positive powers of the braids $x=\sigma_{1} \sigma_{2}^{-1}$ and $y=\sigma_{1}^{2} \sigma_{2}^{-2}$, we see that $g_{i}^{-1} \sigma_{2}^{u} \sigma_{1}$ represents a 1 -positive braid, by Lemma 3.4. Therefore, the braid $g_{i}^{-1} \sigma_{2}^{u} \sigma_{1} w$ is 1-positive, and we conclude that $1<g_{i}<\sigma_{2}^{u} \sigma_{1} w \in C$, hence $g_{i} \in C$ for all $i$, and $C=K_{n}$.

\section{References}

[1] R. Botto Mura and A. Rhemtulla, Orderable Groups, Lecture Notes in Pure and Applied Mathematics, 27 (Marcel Dekker Inc., New York, 1977).

[2] P. Conrad, 'Right-ordered groups', Michigan Math. J. 6 (1959), 267-275.

[3] P. Dehornoy, 'Braid groups and left distributive operations', Trans. Amer. Math. Soc. 345(1) (1994), 115-150.

[4] P. Dehornoy, I. Dynnikov, D. Rolfsen and B. Wiest, Ordering Braids, Surveys and Monographs, 148 (American Mathematical Society, Providence, RI, 2008).

[5] R. Fenn, D. Rolfsen and J. Zhu, 'Centralisers in the braid group and singular braid monoid', Enseign. Math. (2) 42(1-2) (1996), 75-96.

[6] R. C. Lyndon and P. E. Schupp, Combinatorial Group Theory, Classics in Mathematics (Springer, Berlin, 2001), reprint of the 1977 edition.

[7] S. H. McCleary, 'Free lattice-ordered groups represented as $o-2$ transitive $l$-permutation groups', Trans. Amer. Math. Soc. 290(1) (1985), 69-79.

[8] J. Mulholland and D. Rolfsen, 'Local indicability and commutator subgroups of artin groups', Preprint, available via http://arxiv.org/abs/math/0606116.

[9] A. Navas, 'On the dynamics of (left) orderable groups', Ann. Inst. Fourier (Grenoble) 60(5) (2010), $1685-1740$.

[10] A. Navas and B. Wiest, 'Nielsen-Thurston orderings and the space of braid orderings', Preprint, available via http://arxiv.org/pdf/0906.2605.

[11] J. J. Rotman, An Introduction to the Theory of Groups, 4th edn, Graduate Texts in Mathematics, 148 (Springer, New York, 1995).

ADAM CLAY, CIRGET, Université du Québec à Montréal, Case postale 8888,

Succursale Centre-ville, Montréal QC, Canada H3C 3P8

e-mail: aclay@cirget.ca 